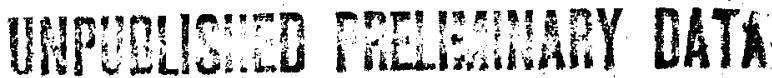

- DEFINITION OF TEMPERATUREIN NONEQUIZIBRIUM PROCESSES
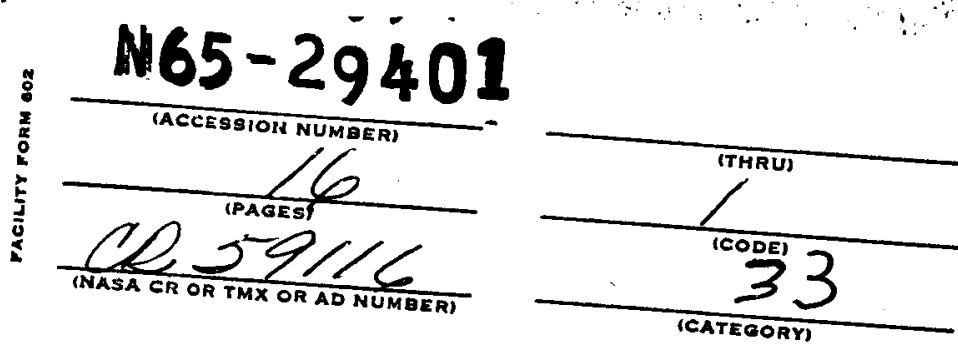

Robert E. Mates and Roger C. Weatherston

Cornell Aeronautical Laboratory, Inc. Buffalo, New York

\section{GPO PRICE CFSTI PRICE(S)}

$\$$

Hard copy $(H C)$

Microfiche $(M F)$

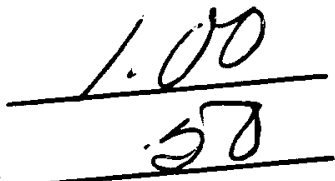

ff 653 July 65

This manuscript has been submitted to

Physics of Fluids for publication.

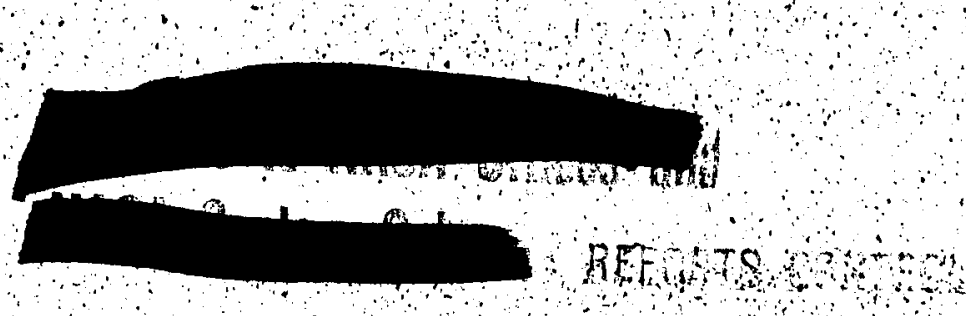




\title{
DEFINITION OF TEMPERATURE IN NONEQUILIBRIUM PROCESSES*
}

\author{
Robert E. Mates ${ }^{* * *}$ and Roger C. Weatherston \\ Cornell Aeronautical Laboratory, Inc. \\ Buffalo, New York
}

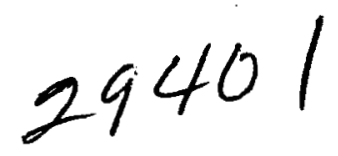

ABSTRACT - This paper examines the validity of applying concepts of temperature as defined in classical thermodynamics to nonequilibrium processes. If the irreversibility in the process can be confined to heat flow between suitably defined subsystems, equilibrium thermodynamics can be applied by the definition of additional temperatures to characterize the subsystems. The classical thermodynamic temperature is defined as suggested by Clausius in terms of heat exchange between a reversible engine and energy reservoirs. It is shown that the above definition of classical thermodynamic temperature can sometimes be applied to a particular degree of freedom, such as vibration, even if it is not in equilibrium with the other degrees of freedom. However, it does not appear possible to extend this concept to a fluid with nonequilibrium chemical composition. To demonstrate this, the entropy rise in vibrational and chemical nonequilibrium processes is computed using the concept of multiple temperatures and compared with the classical result. The results agree for vibrational but not for chemical nonequilibrium.

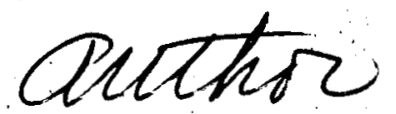

The research reported here was carried out for NASA under Contract No. NASr-109.

*xiseo, State University of New York at Buffalo, Buffalo, New York. 
Introduction

In many problems of current interest involving high temperature, high velocity flows, nonequilibrium phenomena play an important role. These include expansions of high temperature gases in rocket nozzles and hypersonic test facilities, and relaxation phenomena behind shock waves. In analyzing these phenomena it is convenient to employ the methods of classical equilibrium thermodynamics.

While classical thermodynamics is normally applied only to equilibrium systems, some authors have successfully applied classical me thods to nonequilibrium systems by clever definition of the thermodynamic system. A techrique which has been used is to oplit the system into two subsystems, each internally in equilibrium and connected by some irrever sible heat exchange. Kantrowitz ${ }^{1}$ and Connor and Erickson ${ }^{2}$ used this method for analyzing a lagging vibrational degree of freedom, introducing a vibrational temperature to characterize the vibrational subsystem. Wood and Kirkwood ${ }^{3}$ extended the treatment to multiple lagging enexgy modes. Recently Eschenroedex ${ }^{4}$ has applied the same idea to chemical nonequilibrium processes, using a chemical temperature defined by the mass action law. Questions have been raised regarding the validity of these methods. For instance, Heims ${ }^{5}$ has pointed out the limitations of the

'Kantrowitz, A., J. Chem: Phys., 10, 145 (1942).

${ }^{2}$ Connor, L.N. and Erickson, W.D., AIAA J.. 2. 397 (1964).

${ }^{3}$ Wood, W.W. and Kirkwood, J.G., J. App. Phys., 28, 395 (1957).

${ }^{4}$ Eschenroeder, A.Q.; Phys. Fluids, 6. 1408 (1963).

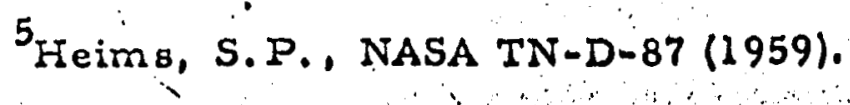


heat flow analogy for the case of simultaneous vibrational and chemical nonequilibrium. The purpose of this paper is to examine the justification for using the concept of multiple temperatures from a classical thermodynamic standpoint.

Thermodynamic temperature is defined classically in terms of a reversible engine operating between two reservoirs, by the relation 6

$$
\frac{T_{1}}{T_{2}}=\frac{Q_{1}}{Q_{2}}
$$

where $T$ is the temperature and $Q$ the amount of heat transferred. Heat may be transferred either by conduction or radiation. Conduction is controlled by the translational energy of the molecules. Radiation occurs through rotational, vibrational or electronic transitions and is thus related to the corresponding energy distributions. On a statistical basis the energy of a gas.is described by a series of distribution functions for the various modes of energy storage. In order to characterize a distribution function by a single parameter, the temperature, each degree of freedom of the gas must be internally equilibrated. ... The statistical description, however, does not require equilibrium between modes of energy storage. Thus we may speak of a translational temperature, a rotational temperature, and so forth. For a gas in complete thermodynamic equilibrium, of course, all these tempertatures are equal. The relationship between the thermodynamic temperature and the various statistical temperatures may be established through the use of a reversible engine.

Consider first a gas which can transfer heat to the engine ondy by conduction. This can be accomplished by making the engines a perfect reflector. If

${ }^{6}$ Zemansky, M.W., Heat and Thermodynamics, 4th Ed., (McGraw-Hill Book Co., Inc., New York, 1957) p. 164. 
the reservoir gas is internally in thermal equilibrium its thermodynamic temperature is given by the translational temperature. Suppose now that one of the modes contributing to radiation is excited by some means without changing the translational temperature. This does not affect the conduction of heat and since this is the only mode of heat transfer allowed, the heat transfer to the engine will be unchanged. Hence, the thermodynamic temperature is still equal to the translational temperature.

Next suppose the gas can transmit or receive heat only by radiation due to vibrational transitions. This can be accomplished by separating the gas from the engine by an evacuated space and making the engine a perfect absorber or black body. Since a gas radiate 8 in spectral lines instead of in a black body distribution it is not obvious that the effective "radiation temperature" is equal to its statistical vibrational temperature. These temperatures may be shown to be the same by the following argument. Suppose first that a gas is brought into complete thermal equilibrium, through radiation, with a black body. At , equilibrium the thermodynamic temperature of the gas and the black body are equal. The thermodynamic temperature of the gas is equal to its translational temperature. However in equilibrium the statistical vibrational temperature is equal to the translational, and hence, thermodynamic temperature. Now suppose the gas is expanded suddenly, lowering its translational temperature without affecting the vibrational energy distribution. If the gas and black body can exchange heat only by radiation the thermal equilibrium will not be disturbed, hence the thermodynamic temperature of the gas as measured by the engine will be the vibrational temperature.

It is apparent from this discussion that the temperature used in classical thermodynamics may be equivalent to any one of several statiotical temperatures 
depending on the particular circumstances. The translational temperature is usually thought of as more fundamental than any of the other s since it is - more easily measured. However practical methods for measuring other temperatures have been developed; for example, the line reversal method for. vibrational temperature in a diatomic gas. ${ }^{7}$ It would appear that any temperature which describes an energy distribution that can exchange heat with the surroundings satisfies the classical definition of a thermodynamic temperature. The use of temperatures other than these cannot be justified by the above arguments. For example, the chemical temperature, as defined above, does not characterize an energy distribution which can exchange heat directly with its surroundings. It should also be noted that for many systems the chemical composition is a function of pressure as well as temperature. For a given chemical composition, which specifies an amount of chemical energy, the chemical temperature cannot be determined unless the pressure or density is also given.

Both Kantrowitz ${ }^{1}$ and Eschenroeder ${ }^{4}$ used the concept of multiple temperatures to calculate the entropy rise in irreveraible adiabatic flows. Kantrowitz considered the irreversibility due to a lagging vibrational heat capacity and obtained

$$
d s=d e_{v}\left(\frac{1}{T_{v}}-\frac{1}{T}\right)
$$

where $T$ is the translational temperature, $T_{v}$ the vibrational temperature,

Fiurle, I.R., Russo, A.I. and Hall, J. Gordon, Spectroscopic Studies of Vibrational Nonequilibrium in Supersonic Nozzle Flows. To be published in. J. Chem. Phys. 
and $d e_{v}$ the amount of energy exchanged between the two modes." Eschenroeder defined the chemical temperature $\tau_{c}$ as that temperature for which a dissociating diatomic gas would be in equilibrium at the same density and degree of dissociation. This model yields

$$
d s=d e_{c}\left(\frac{1}{T_{c}}-\frac{1}{T}\right)
$$

where $d e_{c}$ represents the amount of heat released or absorbed by the chemical reaction.

To resolve the correctness of Eqs. (1) and (2) the entropy rise in nonequilibrium processes is calculated here by choosing a hypothetical reversible path connecting the two end states of the process. It is not always possible to find a reversible process connecting two nonequilibrium states, since in many cases the states cannot be described by a finite number of state variables. The cases considered here are rather special in that each energy distribution is internally in equilibrium. Calculations have been carried out for the two cases described above and are presented in the next two sections. The . expression for vibrational nonequilibrium entropy rise (Eq. (1) ) is verified, but (Eq. (2)) for chemical nonequilibrium does not appear to be valid.

Vibrational Nonequilibrium

Consider a gas in which the vibrational degree of freedom is not equilibrated with the "fast" degyeesoff freedom: translation, rotation, etc. 1 A hypothetical reversible path will be constructed to calculate the entropy rise due to vibrational relaxation. The process is assumed to be free of irreversibility due to other dissipative effects such 28 viscosity. It is assumed that each degree of freedom is internally equilibrated, that is, the energy is distributed according to a Maxwell-Boltzmann distribution law. Then the energy in 
each degree of freedom can be described by a single parameter, the temperature appearing.in the distribution function.

Under these assumptions the internal energy of the gas may be written

$$
e=e_{p}(T)+e_{v}(\alpha, T)
$$

where $e_{f}$ depends only on temperature and $e_{v}$ is a function of temperature and a parameter $\propto$ expressing the degree of vibrational excitation. $T$ is the translational temperature of the gas. In order to compute the change in entropy for an irreversible process on the basis of classical thermodynamics it is necessary to choose some hypothetical reversible path connecting the two end states. On the $T-\rho$ diagram shown in Fig. 1 the real process is denoted by $1-2$. The following reversible path is chosen: (a) The gas is heated or cooled at constant density, keeping the vibrational energy constant, to a temperature $\tau^{\prime}$. $\tau^{\prime}$ is chosen as the temperature at which the gas is in complete thermodynamic equilibrium with the given energy distributions. This process is reversible since no energy exchange occurs between the vibrational mode and the other degrees of freedom. (b) The gas undergoes a reversible process $1^{1}-2$ bringing it to the density and the proper amount of vibrational energy required at state 2. (c) The gas is cooled or heated to state 2 at constant density with frozen vibration.

The hypothetical processes (a) and (c) require special consideration in that their execution requires the vibrational relaxation time to be infinite compared to the time required to maintain translational equilibrium. The fact that no $x$ eal gas could fulfill this requirement does not invalidate the calculation of entropy changes based on such a process. As long as the translational and. vibrational degrees of freedom are each internally, equilibrated (in a Boltzmann 
distribution) the process end states can be described by a set of thermodynamic variables. Hence the entropy, which is a state function, will change by the same amount along any path, reversible or irreversible. The reversible path wa chosen to facilitate the calculation by making use of the equality

$$
\int d s=\int \frac{d Q}{T}
$$

however the result is not dependant on the path. chosen.

The entropy change for the process $1-2$ is the sum of the changes over the path $1-1^{\prime}-2^{\prime}-2$.

$$
\begin{aligned}
& \Delta s_{i^{\prime}}=\int_{1}^{\prime} \frac{d e_{f}}{d T} d T=\int_{T_{1}}^{T^{\prime}} c_{f}(T) \frac{d T}{T} \\
& d s_{1}^{\prime \prime} \dot{2}^{\prime}=\int_{\mu^{\prime}}^{2^{\prime}} \frac{d e_{f}+d e_{r}+\rho d / p}{T} \\
& =\frac{c_{f}\left(T_{1}^{\prime}\right)}{T_{1}^{\prime}} d T_{12^{\prime}}+\frac{d e_{r_{1}^{\prime} 2^{\prime}}}{T_{1}^{\prime}}-\frac{R d p_{12^{\prime}}}{p_{1}^{\prime}} \\
& \Delta S_{2}{ }^{\prime}=-\int_{T_{2}}^{T_{2}^{\prime}} C_{f}(T) \frac{d T}{T} \\
& :=-\int_{T_{1}}^{T_{1}^{\prime}} c_{f}(T) \frac{d T}{T}-c_{p}\left(T_{1}\right) \frac{d T_{12^{\prime}}}{T_{10}}+c_{f}\left(T_{1}\right) \frac{d T_{12}}{T_{1}}
\end{aligned}
$$

Adding the entropy. contributions

$$
\begin{aligned}
d s_{12} & =\frac{d e_{r_{1}{ }^{\prime}}-\frac{R d \rho_{12}}{T_{1}^{\prime}}+c_{1}\left(T_{1}\right) \frac{d T_{12}}{T_{1}}}{\rho_{1}} \\
& =\frac{d e_{v_{12}}-\frac{R d \rho_{1}}{T_{1}}+C_{f}\left(T_{1}\right) \frac{d T_{12}}{T_{1}}}{}
\end{aligned}
$$

8 
An energy balance for the process $1-2$ yields

$$
d g_{12}=C_{p}\left(T_{1}\right) d T_{12}+d e_{r_{12}}-R T_{1} \frac{d p_{12}}{p_{1}}
$$

Hence

$$
d_{s_{12}}=d e_{v_{12}}\left[\frac{1}{T_{1}^{\prime}}-\frac{1}{T_{1}}\right]+\frac{d q_{12}}{T_{1}}
$$

If the actual process is adiabatic the last term of course is zero. This is the exact result obtained in Ref. $I$ and $2, E q .(I)$, and confirms the validity of the use of vibrational temperature in the classical thermodynamic sense.

Chemical Nonequilibrium

Next consider the irreversibility caused by chemical nonequilibrium. Assuming the reactants and products to be ideal gases the internal energy per unit mass of the mixture is given by

$$
e=\alpha e_{p}(T)+(1-\alpha) e_{r}(T)
$$

where $\propto$ is the mass fraction of products and $e_{r}$ and $e_{p}$ are the specific internal energies of reactants and products.

The same path is used $2 s$ in the first case: $\left(1-1^{\prime}\right)$ the gas is heated or cooled at constant density and composition to a temperature $T_{1}^{\prime}$ at which the gas is in chemical equilibrium. $\left(1^{\prime} \times 2^{\prime}\right)$. The gas is brought reversibly to the final density and composition. $(2 !-2)$ " The gas is heated or cooled at constart density and composition to the final temperature. The differential change in internal energy may be written:

$$
\begin{aligned}
d e & \left.\left.=\frac{\partial e}{\partial \alpha}\right)_{T} d \alpha+\frac{\partial e}{\partial T}\right)_{\alpha} d T \\
\quad & =\left(e_{p}-e_{r}\right) d \alpha+c_{v}(\alpha, T) \alpha T
\end{aligned}
$$

9 
where $C_{V}$ is the constant volume specific heat for the mixture.

The entropy contributions are computed as before:

$$
\begin{aligned}
& \Delta s_{11}=\int_{T_{1}}^{T_{1}^{\prime}} \frac{c_{v}\left(\alpha_{1}, T\right)}{T} d T \\
& d s_{1}^{\prime} 2^{\prime}=\frac{d e_{1}^{\prime} 2^{\prime}}{T_{1}^{\prime}}+\frac{p_{1}^{\prime}\left(\alpha / \rho_{1}^{\prime}\right)^{\prime \prime}}{T_{1}^{\prime}} \\
& =\frac{e_{p}\left(T_{1}^{\prime}\right)-c_{r}\left(T_{1}^{\prime}\right)}{T_{1}^{\prime}} d \alpha_{1^{\prime} 2^{\prime}}+C_{v}\left(\alpha_{1}, T_{1}^{\prime}\right) \frac{d T_{1}^{\prime} 2^{\prime}}{T_{1}^{\prime}}-R_{1}^{\prime} \frac{d \rho_{1}^{\prime}{ }^{\prime}}{\rho_{1}^{\prime}}
\end{aligned}
$$

where $R$ is the gas constant for the mixture and varies with composition.

$$
\Delta s_{2^{\prime} 2}=-\int_{T_{1}}^{T_{1}^{\prime}} c_{v}\left(\alpha_{2}, T\right) \frac{d T}{T}-c_{v}\left(\alpha_{2}, T_{1}^{\prime}\right) \frac{d T_{1}^{\prime} 2^{\prime}}{T_{1}^{\prime}}+c_{v}\left(\alpha_{2}, T_{1}\right) \frac{d T_{12}}{T_{1}}(14)
$$

Summing:

$$
\begin{aligned}
d s_{12} & =\int_{T_{1}}^{T_{1}^{\prime}} \frac{C_{v}\left(\alpha_{1}, T\right)-C_{v}\left(\alpha_{2}, T\right)}{T} d T+\frac{\left[C_{v}\left(\alpha_{1}, T_{1}^{\prime}\right)-C_{v}\left(\alpha_{2}, T_{1}^{\prime}\right)\right]}{T_{1}^{\prime} d T_{12^{\prime}}} \\
& +C_{v}\left(\alpha_{2}, T_{1}\right) \frac{d T_{12}}{T_{1}}-R_{1}^{\prime} \frac{d \rho_{12^{\prime}}}{P_{1}^{\prime}}+\left[e_{\mu}\left(T_{1}^{\prime}\right)-e_{r}\left(T_{1}^{\prime}\right)\right] \frac{d \alpha_{1}^{\prime}}{T_{1}^{\prime}}
\end{aligned}
$$

Now

$$
\begin{aligned}
C_{v}\left(\alpha_{1}, T\right)-C_{v}\left(\alpha_{2}, T\right) & =\left[C_{V}(T)-C_{v_{r}}(T)\right]\left(\alpha_{1}-\alpha_{2}\right) \\
& =-\left[C_{v_{p}}(T)-C_{v_{r}}(T)\right] \alpha_{\alpha_{12}}
\end{aligned}
$$

Making these substitutions and neglecting second order differences

$$
\begin{aligned}
d s_{12}= & {\left[-\int_{T_{1}}^{T_{1}^{\prime}} \frac{C_{v_{p}}(T)-C_{v_{r}}(T)}{T} d T\right] d \alpha+C_{r}\left(\alpha, T_{1}\right) \frac{d T_{12}}{T_{1}} } \\
& \quad-R_{1} \frac{d p}{\rho_{1}+\frac{e_{p}\left(T_{1}^{\prime}\right)-e_{r}\left(T_{1}^{\prime}\right)}{T_{1}} d \alpha} d \alpha^{\prime}
\end{aligned}
$$

10 
An cnergy balance for the process $(1-2)$ yields

$$
\frac{d Q_{12}}{T_{1}}=\frac{e_{p}\left(T_{1}\right)-e_{r}\left(T_{1}\right)}{T_{1}} d \alpha+C_{v}\left(\alpha_{1}, T_{1}\right) \frac{d T_{12}}{T^{\prime}}-R_{1} \frac{d p}{\rho_{1}} \ldots
$$

Substituting and rear ranging

$$
\begin{gathered}
d s_{12}=\left[-\int_{T_{1}}^{T_{1}^{\prime}} \frac{C_{v_{p}}(T)-C_{v_{r}}(T)}{T} d T+\frac{e_{p}\left(T_{1}^{\prime}\right)-e_{r}\left(T_{1}^{\prime}\right)}{T_{1}^{\prime}}\right. \\
\left.-\frac{e_{p}\left(T_{1}\right)-e_{r}\left(T_{1}\right)}{T_{1}}\right] d \alpha+\frac{d Q_{12}}{T_{1}}
\end{gathered}
$$

The entropy rise in chemical nonequilibrium processes can also be calculated by the use of the well known Gibbs equation. 8 Equation (18) can be reduced to the Gibbs equation, as shown in the Appendix.

For the particular case of a diatomic gas relaxing adiabatically, $\alpha Q_{12}=0$, $e_{p}=e_{a}$, and $e_{r}=e_{m}$, where $e_{a}$ and $e_{m}$ are the internal energy of the atoms and molecules, respectively. For comparison with Eq. (2) the internal energy of the atoms is redefined to exclude chemical energy

$$
e_{a^{\prime}}=e_{a}-h_{d}^{0}
$$

where $h_{\alpha}^{0}$ represents the dissociation energy at some reference temperat ure. The results may then be written

$$
\begin{aligned}
d s_{12}= & {\left[-\int_{T_{1}}^{T_{1}^{\prime}} \frac{c_{v_{a}}(T)-c_{v_{m}}(T)}{T} d T+\left(\frac{1}{T_{1}^{\prime}}-\frac{1}{T_{1}}\right) h_{\alpha}^{0}\right.} \\
& \left.+\frac{e_{a}\left(T_{1}^{\prime}\right)-e_{m}\left(T_{1}^{\prime}\right)}{T_{1}^{\prime}}-\frac{e_{a}\left(T_{1}\right)-e_{m}\left(T_{1}\right)}{T_{1}}\right] d \alpha
\end{aligned}
$$

${ }^{8}$ Rossini, F.D., Ed., Thermodynamics and Physics of Matter, (Vol, 1, High Speed Aerodynamics and Jet Propulsion, Princeton Univer sity Press, Princeton, New Jersey, 1955) p. 782. 
This is not the same result which is obtained by treating the irreversibility as a heat exchange between two reservoirs (Eq. (2)). This confirms the earliex argument that the chemical temperature defined by the mass action law is not the same 28 the classical thermodynamic temperature defined in terms of a heat engine.

\section{Conclusions}

One method of extending the results of equilibrium thermodynamics to nonequilibrium processes is the use of a heat bath analogy, wherein the ir ever sibility is confined to a heat flow between suitably defined subsystems. This model requires the use of multiple temperatures: The thermodynamic justification for defining these temperatures has been examined by considering the classical definition of thermodynamic temperature. It was found that temperatures characterizing degrees of freedom which are capable of heat exchange satisfy the classical definition, and hence that the heat bath analogy is valid for processes such as rotational or vibrational relaxation as long as each degree of freedom exhibits a Boltzmann distribution. It is not possible to extend the analogy to chemical nonequilibrium using a chemical temperature defined by the mass action law since the temperature introduced does not satisfy. the classical definition.

The entropy rise for vibrational and chemical relaxation has been obtained using equilibrium thermodynamic's and compared with the results of the heat bath analogy. The results agree for vibrational relaxation but disagree . for'chemical relaxation. 


\section{Appendix}

- The Gibbs oquation for a reacting mixture io given by ${ }^{8}$

$$
T d \delta=\alpha E^{\prime}+p \alpha \sim-\sum_{j} \tilde{\mu}_{j} d \eta_{j}
$$

where $\&$, $E$, and $\mathcal{W}$ are the total entropy, internal energy and volume of the mixture, $\tilde{\mu}_{j}$ is the molar chemical potential and $\eta_{j}$ the number of moles. If there are no dissipative effects other than chemical nonequilibrium

$$
d Q=d E+t i d w
$$

Combining Eqo. (20) arid (21) and writing the equation for unit mass of mixture yields

$$
r d s=-\frac{\sum_{j} \mu_{j} \alpha m_{j}}{\sum_{j} m_{j}}+d g .
$$

For a ${ }^{2}$ eacting mixture this may be further simplified by introducing the mass fraction of products $\propto$

$$
T \alpha s=-\left(\mu_{p}-\mu_{\mu}\right) d \alpha+d g
$$

where $\mu_{p}$ and $\mu_{r}$ are the average chemical potentiale for reactantsiand products. For the jth species, the chemical potential at unit pressure is given by

$$
\mu_{j}^{0}=\mu_{j}-R_{j} T \ln p_{j}
$$

Introducing the stoichiometric coefficients $\gamma_{j}^{\prime}$ and $\gamma_{j}^{\prime}$ for reactants and products, Eqs. (22) and (23) yield

$$
d s=-\left[\frac{\mu_{e}^{0}-\mu_{r}^{0}}{T}+\frac{R_{0}}{\sum v_{j} \eta_{j}} \ln \pi_{j} \frac{p_{j}^{p_{j}^{\prime}}}{p_{j} r_{j}}\right] d \alpha+\frac{d g}{T}
$$


wirer $7 \eta_{j}$ is the molecular weight of species $j$. The second term may be rearranged as follows. If $p_{j}^{\prime}$ represents the equilibrium partial pressure at the mixture density and composition, the gas law may be written

$$
p_{j}=p_{j}^{\prime} \frac{T}{T^{\prime}}
$$

where $T^{\prime}$, is the equilibrium temperature for the same density and composition.

Then

$$
\pi \frac{p_{j}^{r_{j}^{\prime}}}{p_{j} r_{j}}=\pi \frac{p_{j}^{\prime} v_{j}^{\prime}}{p_{j}^{\prime} r_{j}}\left(\frac{T}{T_{1}}\right)^{\left(\Sigma v_{j}^{\prime}-\Sigma v_{j}\right)}
$$

Also

$$
\pi \frac{p_{j}^{\prime} v_{j}^{\prime}}{p_{j}^{\prime} v_{j}}=\exp \left\{-\frac{\mu_{p}^{0}\left(T^{\prime}\right)-\mu_{\mu}^{0}\left(T^{\prime}\right)}{R_{0} T^{\prime} / \sum v_{j} z_{j}}\right\} .
$$

The chemical potentials may be eliminated by using

$$
\mu^{0}=e+R T-T s^{\circ} .
$$

Substitutions of Eq. $(25)-(27)$ into $(24)$ gives

$$
\begin{aligned}
\therefore d s= & {\left[-\frac{e_{p}(T)-e_{r}(T)}{T}+\frac{e_{p}\left(T^{\prime}\right)-e_{r}\left(T^{\prime}\right)}{T^{\prime}}+\frac{s_{p}^{0}(T)-s_{r}^{0}(T)}{T}\right.} \\
& \left.-\frac{s_{p}^{0}\left(T^{\prime}\right)-s_{r}^{0}\left(T^{\prime}\right)}{T^{\prime}}-\frac{R_{0}}{\sum_{j} r_{j} m_{j}} \ln \left(\frac{T}{T^{\prime}}\right)^{\left(\Sigma v_{j}^{\prime}-\Sigma v_{j}\right)}\right] d_{\alpha+\frac{d q}{T}}{ }^{(28)}
\end{aligned}
$$

Now

$$
\begin{aligned}
s^{\circ}\left(T^{\prime}\right)-s^{\circ}(T) & \left.=\int_{T}^{T^{\prime}} \frac{\partial s^{\prime}}{\partial T}\right)_{p} d T \\
& =\int_{\gamma}^{T^{\prime}} c_{v} \frac{d T}{T}+R \ln \frac{T^{\prime}}{T}
\end{aligned}
$$

Equations (28) and (29) yield the result

$$
d s=\left[-\int_{T}^{T^{\prime}} \frac{C_{v_{p}}-C_{v_{r}}}{T} d T+\frac{e_{p}\left(T^{\prime}\right)-e_{r}\left(T^{\prime}\right)}{T^{\prime}}-\frac{e_{p}(T)-e_{r}(T)}{T}\right] d \alpha+\frac{d q}{T}(30)
$$

which is identical with Eq. (18)..

14 
